\title{
Primary subglottic malignant melanoma: a case report from Jordan
}

\author{
Qais Aljfout ${ }^{1}$, Hayfa Obaidat ${ }^{2}$, Raed Akayleh ${ }^{2}$, Osama Olwan², Belal Kteshat ${ }^{3}$, Mohammad Ali Hiari ${ }^{2}$
}

${ }^{1} \mathrm{MD}$, Consultant of Otorhinolaryngology, Head \& Neck Surgery, Otorhinolaryngology Department, King Hussein Medical Centre, Royal Medical Services, Amman, Jordan

${ }^{2}$ MD, Otorhinolaryngology Department, King Hussein Medical Centre, Royal Medical Services, Amman, Jordan

${ }^{3}$ MD, Anesthesiology Department, King Hussein Medical Centre, Royal Medical Services, Amman, Jordan

\section{Type of article: Case report}

\begin{abstract}
Primary mucosal melanoma is an under diagnosed and rare tumor. Subglottic malignant melanoma is the least common of mucosal melanoma. This paper reports a case of a 60 -year-old man who presented to the ENT clinic at King Hussein Medical Center in 2015 complaining of shortness of breath and stridor. Fibro optic endoscopic examination revealed a subglottic mass, microlaryngoscopy and biopsy with immunohistochemical staining revealed the diagnosis of malignant melanoma. The patient was treated by surgical excision followed by radiotherapy.
\end{abstract}

Keywords: Larynx, Malignant, Melanoma, Mucosal, Subglottic

\section{Introduction}

Primary malignant melanoma of the larynx are very rare neoplasms (1), they represent $3.6-7.4 \%$ of mucosal malignant melanomas of the upper aerodigestive tract (2-4) and most laryngeal melanomas occur in the supraglottic part of the larynx; almost $60 \%$ of reported cases, and can involve any area of the supraglottic area. Less common sites include the glottis area, true vocal cords and posterior commissure area, up until 1995 there was no documented report of cases involving the subglottic area of the larynx (1). Until now, only few cases of primary subglottic malignant melanoma had been reported (5).

\section{Case presentation}

A 60-year-old gentleman, known to be diabetic and heavy smoker presented to the ENT clinic at King Hussein Medical Center in 2015 with progressive dyspnea of five months duration. He was evaluated many times in the emergency room, a chest x-ray was done and the condition was managed with nebulizers without significant improvement. There was no history of dysphagia, odynophagia, nor obvious neck swelling. Clinical examination and fibro-optic laryngoscopy was done for him in the clinic, which showed large subglottic mass causing more than $50 \%$ obstruction in the subglottic area. The mass was sessile arising from the left anterior subglottic region with dark necrotic tissue around it, both vocal cords were normal and mobile (Figure 1), no palpable cervical lymph nodes were found on clinical examination. Computed tomography scan of neck and chest was done and showed diffuse thickening of the left subglottic region with narrowing of laryngeal lumen at that area, and there was no extension beyond the wall of the larynx. There were multiple small anterior cervical lymph nodes, the largest measured $12 \mathrm{~mm}$ in diameter. No lung lesion, and no hilar or mediastinal lymph node enlargement could be detected (Figure 2). The patient was consented for microlaryngoscopy and excision of the mass was done and sent for histopathology. One week later, the histopathology report was ready with the diagnosis of malignant melanoma. Histopathological sections showed mucosal biopsies infiltrated by a sheet of spindle and epithelioid cells having hyper chromatic nuclei (Figures 3), occasional prominent cherry red nucleus, and abundant eosinophilic cytoplasm with melanin deposit (Figure 4). Although we had suspected malignant lesion and mostly squamous cell carcinoma, the diagnosis

\section{Corresponding author:}

Dr. Qais Aljfout, Otorhinolaryngology Department, King Hussein Medical Centre, Royal Medical Services, Amman, Jordan. Tel.: +962772256809, Email: qaisj@yahoo.com

Received: October 18, 2017, Accepted: December 27, 2017, Published: March 2019

iThenticate screening: December 27, 2017, English editing: April 25, 2018, Quality control: September 11, 2018

(C) 2019 The Authors. This is an open access article under the terms of the Creative Commons Attribution-NonCommercialNoDerivs License, which permits use and distribution in any medium, provided the original work is properly cited, the use is non-commercial and no modifications or adaptations are made. 
of malignant melanoma was unexpected for us; we had consulted our dermatology colleagues for proper evaluation, PET/CT scan failed to reveal other primary source or reveal that there was no distant metastasis. So the patient was diagnosed with primary subglottic malignant melanoma, and treatment was completed by radiotherapy.

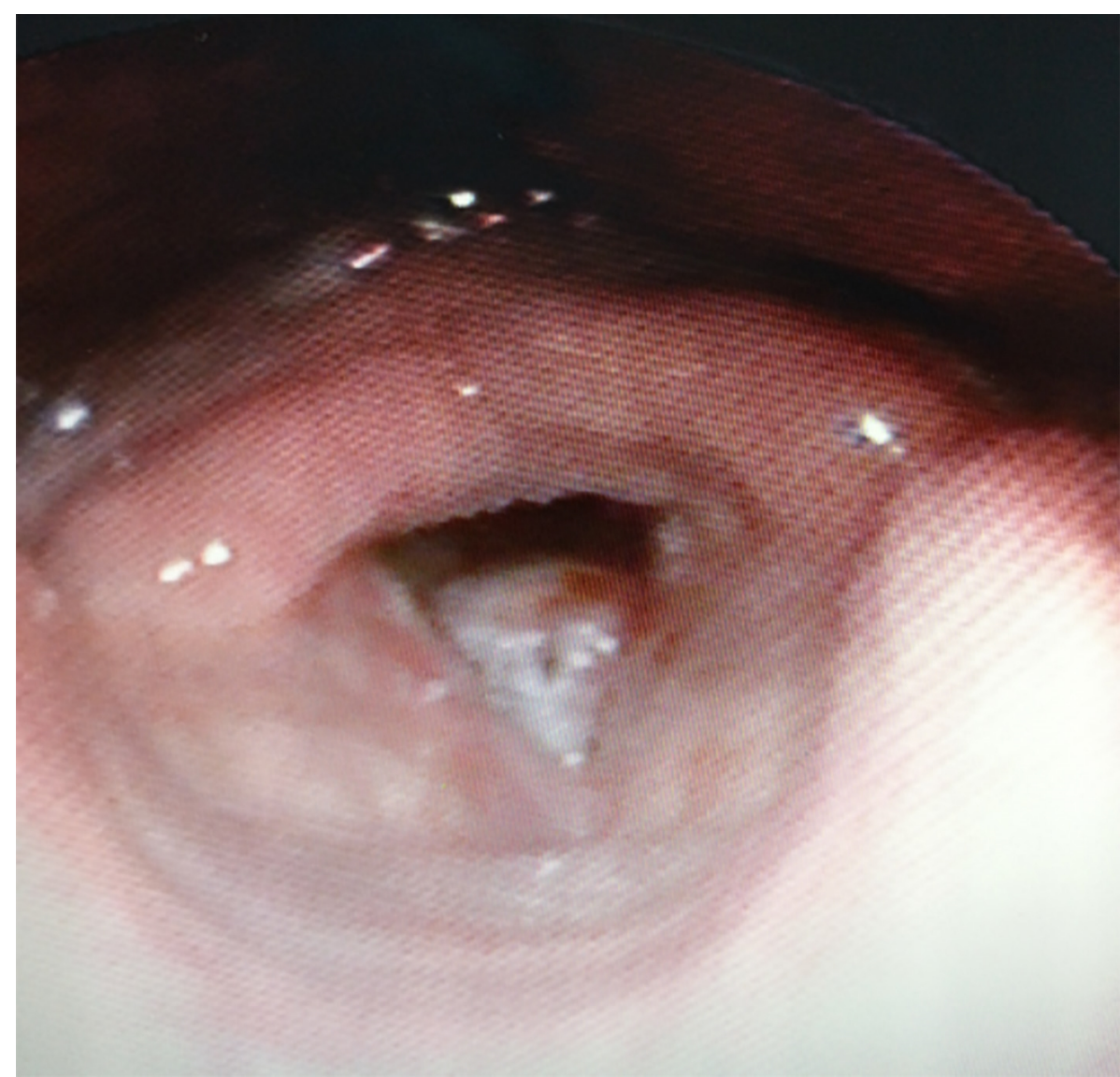

Figure 1. Endoscopic view of the subglottic mass in the clinic.

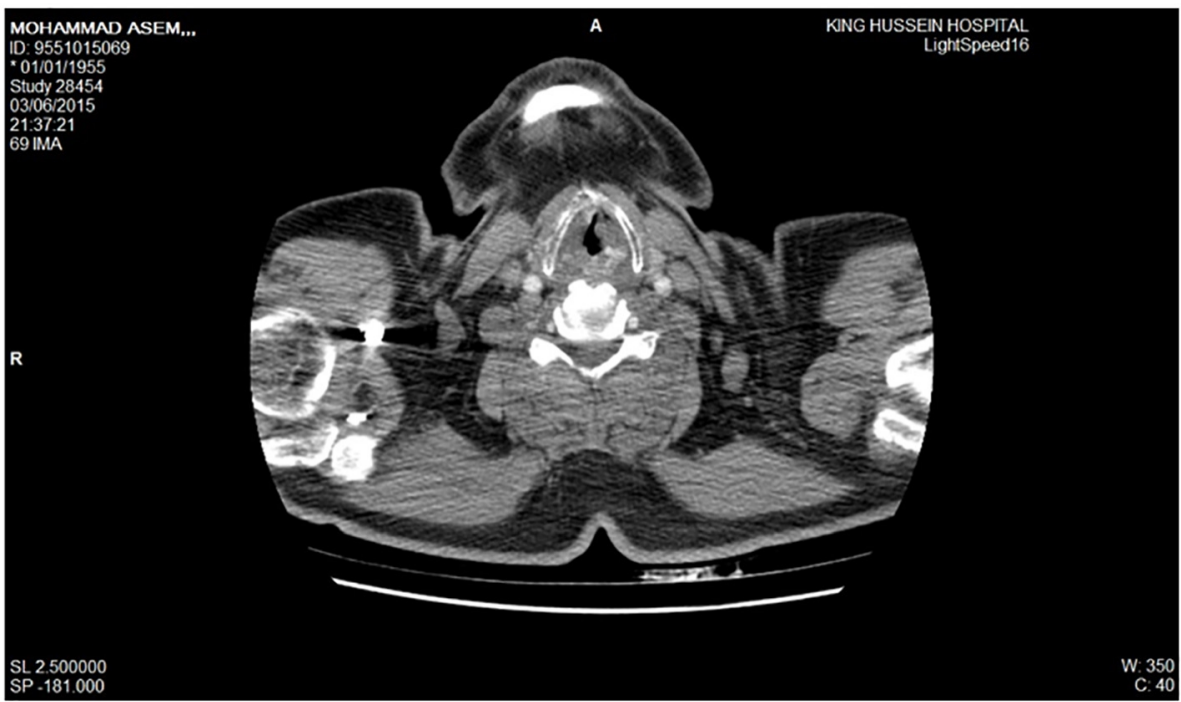

Figure 2. CT scan section showing the subglottic soft tissue density. 


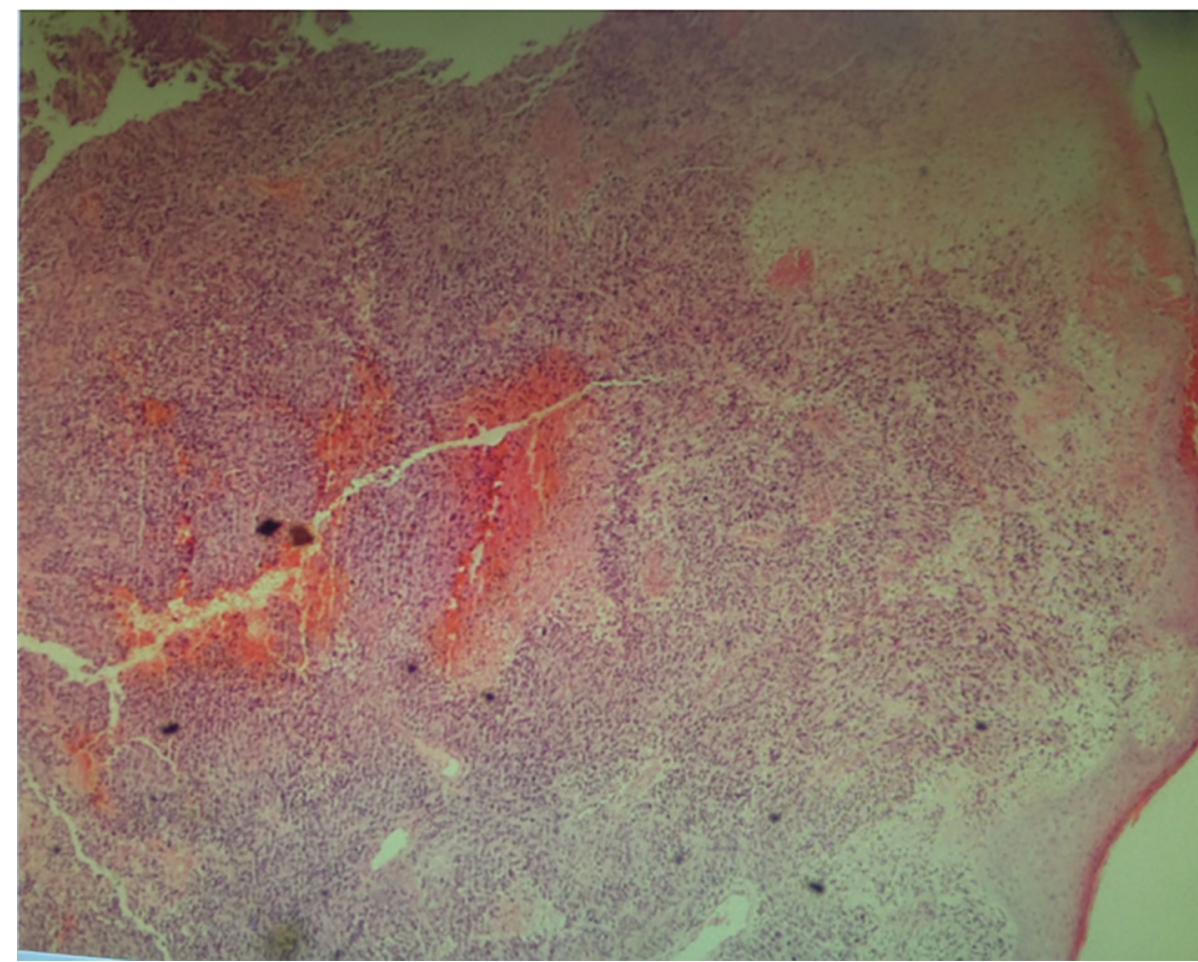

Figure 3. H\&E stain of biopsy (stained 40X). The tumor cells are immunoreactive for melanin A, vimentin and S 100

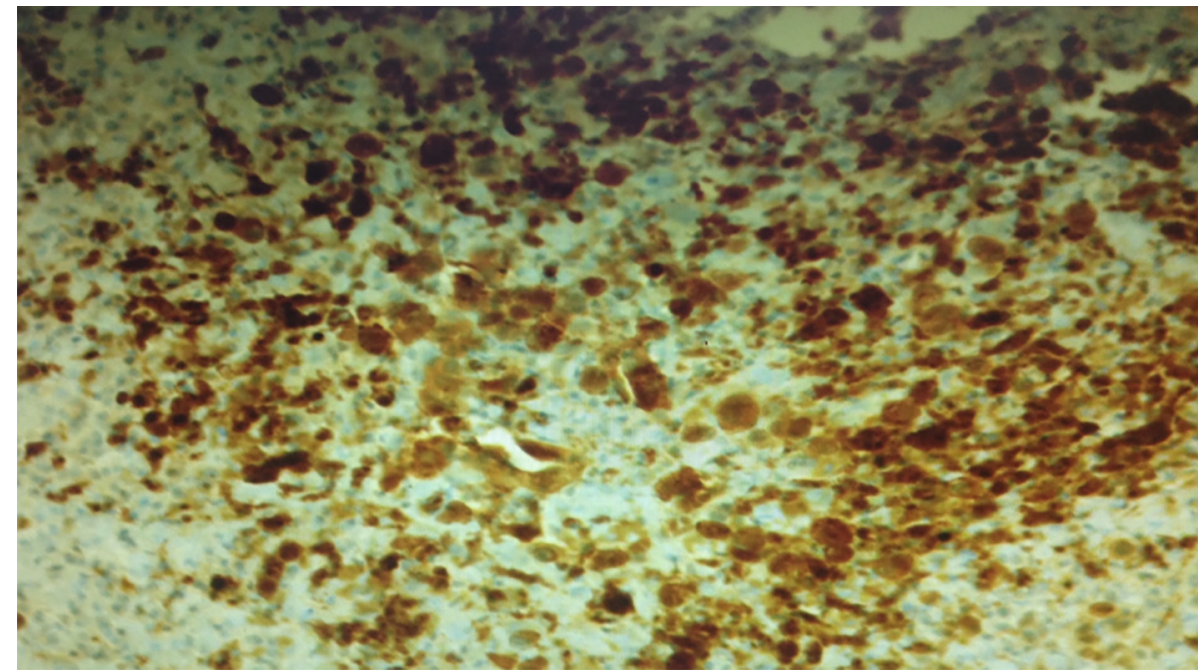

Figure 4. Immunohistochemical staining of the biopsy (stained 40X).

\section{Discussion}

Malignant melanomas are often cutaneous in origin, and they have a low incidence rate. Mucosal malignant melanomas consist of $0.5-3 \%$ of all malignant melanomas $(6,7)$. Although the number of cases reported is small, smoking is considered to be a major risk factor as other malignant laryngeal tumors $(6,7)$ and other studies have linked it to human papilloma virus, sun light exposure and other carcinogens $(6,8)$. Similar to other malignant melanomas, it had been linked to an altered immune system and connected to a few genes such as IL17A and CD70 (6). Primary laryngeal melanomas develop from neural crest derived melanocytes that had migrated to the mucosa of the larynx as well as the mucosa of upper aerodigestive tract (9) and this can be supported by the presence of intralaryngeal nevi (10), melanin pigmentation of the larynx (11) and lentigo of the larynx (12). Although hoarseness and shortness of breath are the common presenting symptoms associated with sore throat, dysphagia, neck pain and referred ear pain, in our case; the patient presented with shortness of breath and his voice was normal 
due to the subglottic origin and this might have attributed to delay of diagnosis despite frequent visits to the emergency room, This is one of the challenges in the diagnosis of such tumors. The other challenge is to differentiate primary from metastatic secondary tumors, because it is reported that malignant melanoma might disappear from its primary site after metastasis (13). One clue for the diagnosis on endoscopic evaluation is the presence of brown or black pigmentations (13) and in our case; this was misinterpreted as areas of necrosis. Firm diagnosis of malignant melanomas is based on histopathological examination of tissues, hematoxylin and eosin staining show epithelioid, spindle shaped pleomorphic malignant cells that contain dark brown cytoplasmic and nuclear melanin (14), and immunohistochemical staining detects melanin markers as HMB-45, S-100, PNL2 and Melan-A. Melanoma stage II may be identified under electron microscopy (14). Multiple imaging modalities which include FDGPET/ CT have important roles in the proper evaluation of the primary tumour, assessment of metastatic disease and monitoring the response to treatment (15). Complete surgical excision is the treatment of choice for laryngeal malignant melanomas, and incidence of regional lymph nodes metastasis is considered low (13), but mucosal malignant melanomas of the head and neck tend to present with more invasive disease than cutaneous ones, so more metastatic disease through vascular invasion was reported as up to $80 \%$ (16). Post-operative radiotherapy was reported to improve local control (17) and until now, chemotherapy is not recommended as part of management (7). Despite the fact that laryngeal malignant melanomas have lower incidence of recurrence in comparison to other mucosal melanomas, they have worse behavior. The prognosis is extremely poor with reported survival to be less than 3.5 years $(16)$.

\section{Conclusions}

Although primary mucosal melanomas of the larynx are rare tumors, it should be suspected in patients who have laryngeal mass with surface discoloration. Imunohistochemical staining has an important role in the diagnosis. Although prognosis is poor, surgical excision and radiotherapy are the corner stones for treatment.

\section{Acknowledgments:}

We would like to thank our residents in the department for their help in collecting data and materials. The study received no funds from any resource.

\section{Conflict of Interest:}

There is no conflict of interest to be declared.

\section{Authors' contributions:}

All authors contributed to this project and article equally. All authors read and approved the final manuscript.

\section{References:}

1) Wenig BM. Laryngeal mucosal malignant melanoma. A clinicopathologic, immunohistochemical, and ultrastructural study of four patients and a review of the literature. Cancer. 1995; 75(7): 1568-77. doi: 10.1002/1097-0142(19950401)75:7<1568::AID-CNCR2820750704>3.0.CO;2-M.

2) Moore ES, Martin H. Melanoma of the upper respiratory tract and oral cavity. Cancer. 1955; 8: 1167-76. doi: 10.1002/1097-0142(1955)8:6<1167::AID-CNCR2820080613>3.0.CO;2-I.

3) Nsamba C. A case of malignant melanoma of the larynx. J Laryngol Otol. 1966; 80: 1178-81. doi: 10.1017/S0022215100066536. PMID: 5927751.

4) Cady B, Rippey JH, Frazell EL. Non-epidermoid cancer of the larynx. Ann Surg. 1968; 167: 116-20. doi: 10.1097/00000658-196801000-00019. PMID: 5635178, PMCID: PMC1387226.

5) Ahmad S, Abdelghany M, Goldblatt C, Stark O, Masciotra N. A case of primary subglottic malignant melanoma with a successful surgical treatment. Case Rep Oncol Med. 2014; 2014: 968926. doi: $10.1155 / 2014 / 968926$.

6) Sirikanjanapong S, Lanson B, Amin M, Martiniuk F, Kamino H, Wang BY. Collision tumor of primary laryngealmucosal melanoma and invasive squamous cell carcinomawith IL-17A and CD70 gene overexpression. Head Neck Pathol. 2010; 4(4): 295-9. doi: 10.1007/s12105-010-0200-8. PMID: 20697851, PMCID: PMC2996505.

7) Chang AE. Karnell LH. Menck HR. The National Cancer Data Base report on cutaneous and noncutaneous melanoma. Cancer. 1998; 83(8): 1664-78. doi: 10.1002/(SICI)1097-0142(19981015)83:8<1664::AIDCNCR23>3.0.CO;2-G.

8) Wagner M, Morris CG, Werning JW, Mendenhall WM. Mucosal melanoma of the head and neck. The American Journal of Clinical Oncology. 2008; 31(1): 43-8. 
9) Busuttil A. Dendritic pigmented cells within the human laryngeal mucosa. Arch Otolaryngol. 1976; 102: 43-4. doi: 10.1001/archotol.1976.00780060089012. PMID: 1247414.

10) Wenig BM. Malignant melanoma. Neoplasms of the larynx. Edinburgh: Churchill Livingstone. 1993: 207 30.

11) Pesce C, Toncini C. Melanin pigmentation of the larynx. Acta otoravngor. 1983; 96-2.

12) Travis LW, Sutherland C. Coexisting lentigo of the larynx and melanoma of the oral cavity: report of a case. Otolaryngol Head Neck Surg. 1980; 88: 218-20. doi: 10.1177/019459988008800304.

13) Amin HM, Petruzzelli GJ, Husain AN, Nickoloff BJ. Primary malignant melanoma of the larynx. Arch Pathol Lab Med. 2001; 125(2): 271-3. doi: 10.1043/0003-9985(2001)125<0271:PMMOTL >2.0.CO;2. PMID: 11175650.

14) Durai A, Hashmi S. Primary malignant melanoma of the epiglottis: a rare presentation. Ear Nose Throat J. 2006; 85(4): 274-7. PMID: 16696365.

15) Keraliya AR, Krajewski KM, Braschi-Amirfarzan M, Tirumani SH, Shinagare AB, Jagannathan JP, et al. Extracutaneous melanomas: a primer for the radiologist. Insights Imaging. 2015; 6: 707-17. doi: 10.1007/s13244-015-0427-8. PMID: 26334521, PMCID: PMC4656230.

16) Reuter VE, Woodruff JM. Melanoma of the larynx. Laryngoscope. 1986; 94: 389-93. doi: 10.1288/00005537-198604000-00010.

17) Saigal K, Weed DT, Reis IM, Markoe AM, Wolfson AH, Nguyen-Sperry J. Mucosal melanomas of the head and neck: the role of postoperative radiation therapy. ISRN Oncology. 2012; (2012). 